\title{
PROPIEDADES QUÍMICAS, COLOR Y HUMECTABILIDAD DE PARTÍCULAS DE Laureliopsis philippiana (TEPA) CON Y SIN TRATAMIENTO TÉRMICO
}

\author{
CHEMICAL PROPERTIES, COLOR AND WETTABILITY OF \\ Laureliopsis philippiana (TEPA) PARTICLES WITH AND WITHOUT \\ THERMAL TREATMENT
}

\author{
Rommel Crespo G ${ }^{1,2}$, Marcos Torres U. ${ }^{1}$, Luis Valenzuela H. ${ }^{3}$, Hernán Poblete W. 1,^
}

\begin{abstract}
RESUMEN
El propósito de este trabajo fue determinar el efecto de un tratamiento térmico, en una atmósfera saturada de vapor de agua, en partículas de madera de la especie Laureliopsis philippiana. Las partículas fueron tratadas en autoclave a $150^{\circ} \mathrm{C}$ por 90 minutos y a una presión de $430 \mathrm{kPa}$, la cual se generó durante el proceso por la evaporación del agua en el autoclave herméticamente cerrado. Se determinaron la cantidad de extraíbles en soda, etanol tolueno, agua caliente y fría, así también, los porcentaje de celulosa, holocelulosa y lignina, el valor $\mathrm{pH}$, los porcentajes de ácidos volátiles y solubles en agua, y la capacidad tampón, tanto para partículas sin tratamiento como con tratamiento. Para las partículas con tratamiento térmico, se encontró un aumento en el porcentaje de extraíbles, de celulosa, y de ácidos volátiles y lavables, así como también, un aumento de la acidez y de una mayor capacidad tampón, en comparación con las partículas sin tratamiento. El tratamiento térmico produjo un cambio de color en las partículas y una reducción de la humectabilidad. Debido a los cambios químicos encontrados en las partículas tratadas, estas presentarían ventajas en la fabricación de tableros de partículas, favoreciendo el fraguado de la ureaformaldehído.
\end{abstract}

Palabras claves: Composición química, acidez, Laureliopsis philippiana, tratamiento térmico, tableros de partículas.

\begin{abstract}
The purpose of this work was to determine the effect of a thermal treatment in a vapor water saturated atmosphere, on wood particles of Laureliopsis philippiana. The particles in autoclave at $150^{\circ} \mathrm{C}$ for 90 minutes and with a pressure of $430 \mathrm{kPa}$ were thermally treated, the pressure generated by the evaporation of water in the sealed autoclave. The amount of extractives in soda, ethanol-toluene, cold and hot water, as well as, the percentages of cellulose, holocellulose and lignin, the $\mathrm{pH}$ value, the percentages of volatiles and washables acids, and buffer capacity, for both particles without and with treatment, were determined. In thermally treated particles an increase in the percentage of extractives, cellulose and volatiles and water soluble acids was found, as well as, in thermally treated particles an increment of acidity and a higher buffer capacity was found, in comparison with the particles without treatment. The thermal treatment produced a color change in the particles and a reduction in their wettability. Due to the chemical changes found in the treated particles they could present advantages in the particleboard production, allowing a better ureaformaldehyde polymerization.
\end{abstract}

Keywords: Chemical composition, acidity, Laureliopsis philippiana, thermal treatment, particleboards.

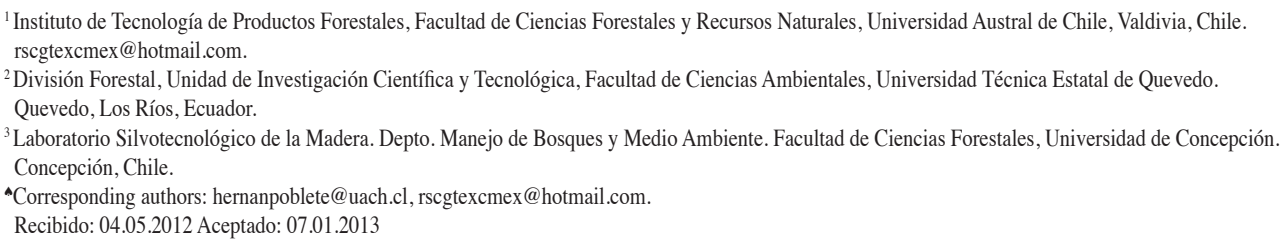




\section{INTRODUCCIÓN}

Un tratamiento térmico en la madera, mejora su estabilidad dimensional y su resistencia contra la biodegradación por hongos de la pudrición, y reduce algunas de sus propiedades mecánicas (Kocaefe et al. 2010). Un tratamiento térmico a altas temperaturas (mayores a $170^{\circ} \mathrm{C}$ ) produce cambios químicos de los constituyentes de la pared celular (hemicelulosa, celulosa y lignina) (Niemz et al. 2004, Metsä-Kortelainen y Viitanen 2012), y puede mejorar la estabilidad dimensional de la matriz celular y con ello, la de las partículas y fibras de madera que conforman un tablero (Boonstra et al. 2006a).

Paralelamente, en muchos de los tratamientos térmicos en autoclave se genera presión, la cual tiene efectos que pueden ser importantes sobre la composición, estructura y propiedades de la madera (Giebeler 1983).

Considerando la importancia de los tratamientos térmicos, así como también, su efecto en las propiedades de la madera, y por ende, su incidencia potencial en la producción de tableros, el objetivo de este trabajo fue: determinar el efecto de un tratamiento térmico en una atmósfera saturada en vapor de agua, en partículas de madera. En este estudio se utilizaron partículas de madera de Laureliopsis philippiana, especie con características químicas desfavorables para la producción de tableros con ureaformaldehído (Poblete y Pinto 1993). Se reportó el efecto en las propiedades químicas y físicas que afectan la producción de tableros elaborados con partículas de madera de $L$. philippiana, encolados con ureaformaldehído, considerando los cambios en los compuestos principales de la pared celular, el porcentaje de extraíbles, ácidos volátiles y lavables, así como, la capacidad tampón y la variación del valor $\mathrm{pH}$. En relación con las propiedades físicas de las partículas, se evaluó el cambio de color y la humectabilidad con ureaformaldehído.

\section{Efecto de la temperatura en los componentes de la madera}

La madera cuando es sometida a la acción del calor está sujeta a variaciones físico-químicas. Los primeros cambios físico-químicos surgen desde los $50{ }^{\circ} \mathrm{C}$ (Schaffer 1973). En muchos tipos de procesamiento de la madera, esta es sometida a temperaturas sobre $250{ }^{\circ} \mathrm{C}$ lo que influye en las propiedades físicas, estructurales y químicas, produciéndose una degradación térmica (Fengel y Wegener 1983). No solo el nivel de la temperatura incide en la degradación térmica de la madera, también se combinan el tiempo de exposición, el tipo de atmósfera, la presión utilizada, y el contenido y distribución del agua en la madera. Así, dependiendo de las condiciones térmicas, los cambios en la madera pueden ser observados a partir de $100^{\circ} \mathrm{C}$ (Fengel y Wegener 1983).

Con temperaturas superiores a $65{ }^{\circ} \mathrm{C}$ y durante períodos prolongados de tiempo, se han detectado efectos irreversibles en la madera, una incipiente degradación térmica de la pared celular. Generalmente, se produce una reducción del grado de polimerización de la celulosa y cambios químicos en las hemicelulosas. De acuerdo con Bekhta y Marutzky (2007) la estabilidad de la celulosa y lignina dependen de la temperatura, disminuyendo con un aumento de la temperatura. Figueroa y Moraes (2009), mencionan que los polímeros de la madera presentan estabilidad térmica diferenciada en función de la red cristalina de cada uno.

De acuerdo con Haygreen y Bowyer (1982) y Bekhta et al. (2003) con temperaturas mayores a $100{ }^{\circ} \mathrm{C}$ en la madera se inicia una descomposición en sus elementos químicos constituyentes (carbono, hidrógeno y nitrógeno). Entre 100 y $200{ }^{\circ} \mathrm{C}$ la madera se degradará gradualmente, el vapor de agua es expulsado junto con dióxido y monóxido de carbono (Haygreen y Bowyer 1982) y vestigios de productos biológicos (Figueroa y Moraes 2009).

Durante la termólisis se provoca la formación de ácido acético y fórmico, los cuales se generan por la ruptura de los grupos acetilo (Poblete 1983, Tjeerdsma et al. 1998). Esos ácidos son importantes como catalizadores en las reacciones que ocurren durante la termólisis, y participan en las reacciones de división, hidrólisis, de la celulosa y hemicelulosa (Tjeerdsma et al. 1998), siendo la hidrólisis sólo destacable en hemicelulosas, mientras que en la celulosa la hidrólisis es limitada (Martín-Sampedro et al. 2011b) 


\section{Efecto de la temperatura en la acidez}

El valor de $\mathrm{pH}$ en una especie está dado por la mayor o menor cantidad de ácidos presentes como compuestos extraíbles. Los tratamientos térmicos provocan una descomposición de la hemicelulosa por hidrólisis, formándose ácidos orgánicos que acidifican la madera (Poblete 1983, Kocaefe et al. 2007).

La acidez es un factor importante a considerar en la fabricación de tableros de partículas. La madera de $L$. philippiana registra altos valores de $\mathrm{pH}$, lo que dificulta el fraguado de la ureaformaldehído (Poblete y Pinto 1993). Las reacciones químicas que ocurren durante el fraguado de los adhesivos siguen su curso óptimo con un rango de $\mathrm{pH}$ específico para cada tipo de adhesivo y su variación afecta las propiedades de la unión entre las partículas. El conocimiento de la acidez de la madera y sus cambios por la forma de procesamiento, son importantes para predecir el comportamiento de las uniones madera- adhesivo.

\section{Efecto del tratamiento en el color}

Tanto los tratamientos térmicos con vapor de agua como los realizados en una atmósfera seca y sin oxígeno, producen cambios de color en la madera (Poblete et al. 2005, Bächle et al. 2010). Con la higrotermólisis (tratamiento en atmósfera con vapor de agua) el cambio de color es más intenso que con un tratamiento en atmósfera sin agua (Boonstra et al. 2006b).

De acuerdo con Boonstra et al. (2006b) un incremento de la temperatura de tratamiento, cambia el color a un tono más oscuro. El cambio de color también depende de la madera usada y se correlaciona con su densidad, al aumentar la densidad la tonalidad se hace más oscura.

Los cambios químicos, provocados por el tratamiento térmico, contribuyen al cambio de color de las partículas de madera. La condensación de las moléculas de tanino, la oxidación de los grupos hidroxilos en moléculas flavonoides (Yongming et al. 2010), la formación de productos de la degradación de los polímeros de la madera (Sundqvist y Morén 2002), la formación de quinonas por la caramelización de las holocelulosas (Tjeerdsma et al. 1998, Boonstra y Tjeerdsma 2006), y la formación de productos de condensación y oxidación de la lignina (Yao et al. 2012) provocan el cambio de color. Yongming et al. (2010) mencionan que al eliminar los extraíbles se puede disminuir la variación de los parámetros de color.

El color de la madera es una propiedad crucial para el consumidor final. La tonalidad oscura de la madera es a menudo favorecida, debido a la función decorativa (Yongming et al. 2010). Sin embargo, en el caso de la fabricación de tableros, se prefiere la obtención de superficies claras que faciliten la posterior terminación del producto con recubrimientos.

\section{Efecto del tratamiento en la humectabilidad}

La madera es un material higroscópico debido a la presencia de grupos hidroxilos. Muchas de las propiedades de la madera dependen de estos grupos y en particular, de las interacciones de las uniones de hidrógeno entre los polisacáridos de la pared celular.

Con la degradación de los carbohidratos después del tratamiento térmico, la concentración de los grupos hidroxilos absorbiendo agua disminuye, resultando en una disminución de la captación de agua y absorción. La despolimerización de los carbohidratos, especialmente de la hemicelulosa, trae consigo una reducción de la cantidad total de grupos hidroxilos, incluyendo los grupos hidroxilos libres. La madera térmicamente modificada absorbe menos agua que las muestras no tratadas. Cuanto mayor sea la temperatura y el tiempo de exposición en un tratamiento térmico, menor será la cantidad de agua que absorbe la madera (Poblete 1983, Kartal et al. 2007). Sin embargo, Martín-Sampedro et al. (2011b) al someter astillas de Eucalyptus globulus no hidratadas e hidratadas, a un tratamiento de explosión con vapor $\left(183{ }^{\circ} \mathrm{C} / 5-10 \mathrm{~min}\right)$ a una presión de $0,98 \mathrm{MPa}$ y descargo de $0,50 \mathrm{MPa}$, informaron de una mayor capacidad de retención de agua, a pesar de la disminución del contenido de hemicelulosa. 
Hakkou et al. (2005), señalan que las reacciones de degradación y la generación de extraíbles durante el tratamiento térmico no son el origen de las propiedades hidrofóbicas de la madera. El cambio de la humectabilidad puede explicarse por una modificación del arreglo conformacional de los polisacáridos, ya que la cristalinidad de la celulosa incrementa con la temperatura debido a la degradación de la celulosa amorfa, la fácil degradación de las hemicelulosas y, probablemente, a la plastificación de la lignina.

La propiedad de la superficie de la madera de absorber agua u otros líquidos tiene un efecto en su pintabilidad y capacidad de encolado (Petric et al. 2007, Metsä-Kortelainen y Viitanen 2012). Esta propiedad es importante cuando se desea obtener un producto, en el cual, se emplea un adhesivo contenido en un solvente acuoso, como es en el caso de los tableros a base de madera.

\section{Efecto de la presión}

De acuerdo con los resultados de Giebeler (1983) los cambios en la presión aplicada durante el tratamiento térmico provocan variaciones en la pérdida de masa y en la estabilidad dimensional de la madera. Un aumento de la presión (de 200 a $1000 \mathrm{kPa}$ ) causa una pérdida de masa, un incremento de la estabilidad dimensional y disminución de las propiedades mecánicas. Por esta razón, al aplicar un tratamiento térmico la presión debe ser controlada.

\section{MATERIALES Y MÉTODOS}

\section{Materiales}

Se utilizó madera de Laureliopsis philippiana Losser, cosechada en la XIV Región de Los Ríos pre cordillera andina (Chile).

\section{Metodología}

-Obtención de partículas

La madera fue viruteada y secada a $45^{\circ} \mathrm{C}$ hasta un contenido de humedad del $4 \%$.

\section{-Tratamiento térmico (higrotérmico)}

Las partículas fueron colocadas en una cesta de acero en autoclave a $150^{\circ} \mathrm{C}$ por 90 minutos. El autoclave contenía agua hasta una altura que dejaba un espacio de $2 \mathrm{~cm}$ entre partículas y agua. Debido al vapor de agua generado en el autoclave, se produjo un aumento de la presión durante el proceso, la cual fue controlada y alcanzó a $430 \mathrm{kPa}$. Finalizado el tratamiento, las partículas se climatizaron a humedad y temperatura ambiente.

\section{-Determinación del color}

La caracterización de la coloración de las partículas antes y después del tratamiento se realizó empleando las tablas de colores para suelos (Munsell 1994). Se empleó también un espectrofotómetro X-Rite de medición de color. Se realizaron mediciones con la opción de brillo incluido, con iluminante D65/10. Durante el proceso de medición, se utilizó un software incorporado en el equipo.

\section{-Humectabilidad}

Se midió la capacidad de absorción de agua determinando la variación del ángulo de la gota con la superficie de la partícula (Poblete 1983). Para este efecto, se ubicaron las partículas en un pedestal, depositándose una gota de adhesivo (ureaformaldehído al $65 \%$ de sólidos) y empleando una cámara fotográfica se obtuvieron fotografías cada 30 segundos, por un lapso de seis minutos. Posteriormente, las fotografías fueron analizadas digitalmente determinándose el ángulo de la tangente de la gota con la superficie de la partícula y su variación en el tiempo, como se puede apreciar en la figura 1 . 


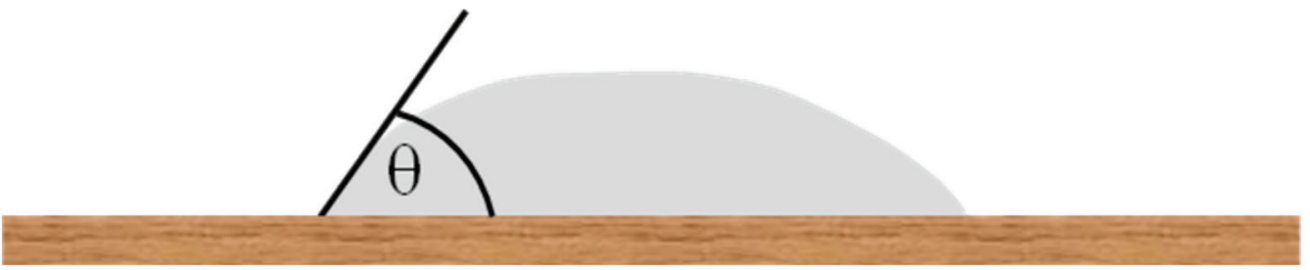

Figura 1. Humectabilidad: Determinación del ángulo $(\theta)$ formado entre la gota de ureaformaldehído ( $65 \%$ de sólidos) y la partícula de madera de L. philippiana.

\section{-Análisis químicos}

La determinación de las propiedades químicas se realizó de acuerdo a las normas y métodos que se especifican a continuación:

- Solubilidad en agua de madera y pulpa (TAPPI $207 \mathrm{~cm}-99$ ).

- Solubilidad en $1 \%$ de hidróxido de sodio $(0,25 \mathrm{~N})$ de madera y pulpa (TAPPI 212 om-98).

- Extraíbles en etanol tolueno (TAPPI $204 \mathrm{~cm}-97$ ).

- Preparación de la madera para análisis químico (TAPPI $264 \mathrm{~cm}-97$ ).

- Celulosa método de Kurschner y Hoffer, según Hessler y Merola (1949).

- Holocelulosa método de Poljak, según Rodríguez (1978).

- Lignina insoluble en ácido (lignina Klason) (TAPPI 222 om-98).

- Valor de pH en el filtrado de una solución de aserrín con agua, según Roffael et al. (2000).

- Ácidos volátiles y solubles en agua, según Poblete (1983).

- Capacidad tampón, según Roffael et al. (2000).

\section{RESULTADOS Y DISCUSIÓN}

\section{Color}

Se detectó un cambio de color en las partículas de amarillo pálido (2,5 Y 8/2) a café (7,5 YR 5/4), según la comparación realizada con las tablas de colores para suelos Munsell. Los resultados obtenidos de la medición en el espectrofotómetro indican que las partículas tratadas térmicamente son más oscuras (menor valor de $L$ ) y menos amarillas (menor valor de b) (tabla 1).

Tabla 1. Comparación del color en partículas de L. philippiana no tratadas y tratadas térmicamente .

\begin{tabular}{|c|c|c|}
\hline Parámetro & $\begin{array}{c}\text { L. philippiana } \text { sin } \\
\text { tratamiento }\end{array}$ & $\begin{array}{c}\text { L. philippiana con } \\
\text { tratamiento }\end{array}$ \\
\hline$L$ (blancura) & 61,78 & 46,55 \\
\hline$a$ (escala de rojo a verde) & 8,88 & 8,88 \\
\hline$b$ (cromaticidad de amarillo a azul) & 25,61 & 20,13 \\
\hline
\end{tabular}


El cambio de color observado en este experimento, concuerda con lo detectado por diferentes autores, quienes encontraron cambios de color de claro a oscuro luego de un tratamiento térmico en vapor de agua y presión (Poblete et al. 2005), en agua y vapor de agua bajo presión seguido de un secado y curado (Boonstra et al. 2006b), y en un horno sin oxígeno (Bächle et al. 2010).

El cambio de color en las partículas de L. philippiana tratadas térmicamente, podría deberse al aumento de la cantidad de extraíbles, como se puede observar en la tabla 2. En menor grado podrían contribuir al cambio de color la caramelización de los productos fraccionados provenientes de la relativa despolimerización de la hemicelulosa (Boonstra y Tjeerdsma 2006), la formación de quinonas (Tjeerdsma et al. 1998) y por la rotura de los enlaces éter $\beta-O-4$ en la estructura de la lignina (Martín-Sampedro et al. 2011a).

Considerando que el mercado de mueble y la construcción, prefieren tableros con superficies claras, el oscurecimiento de las partículas puede traducirse en una desventaja para la comercialización de tableros fabricados con madera tratada térmicamente.

\section{Humectabilidad}

La variación de los ángulos de contacto medidos con respecto a un tiempo de seis minutos, entre la gota de adhesivo ureaformaldehído y las superficies de las partículas tratadas y no tratadas, se muestran en la figura 2.

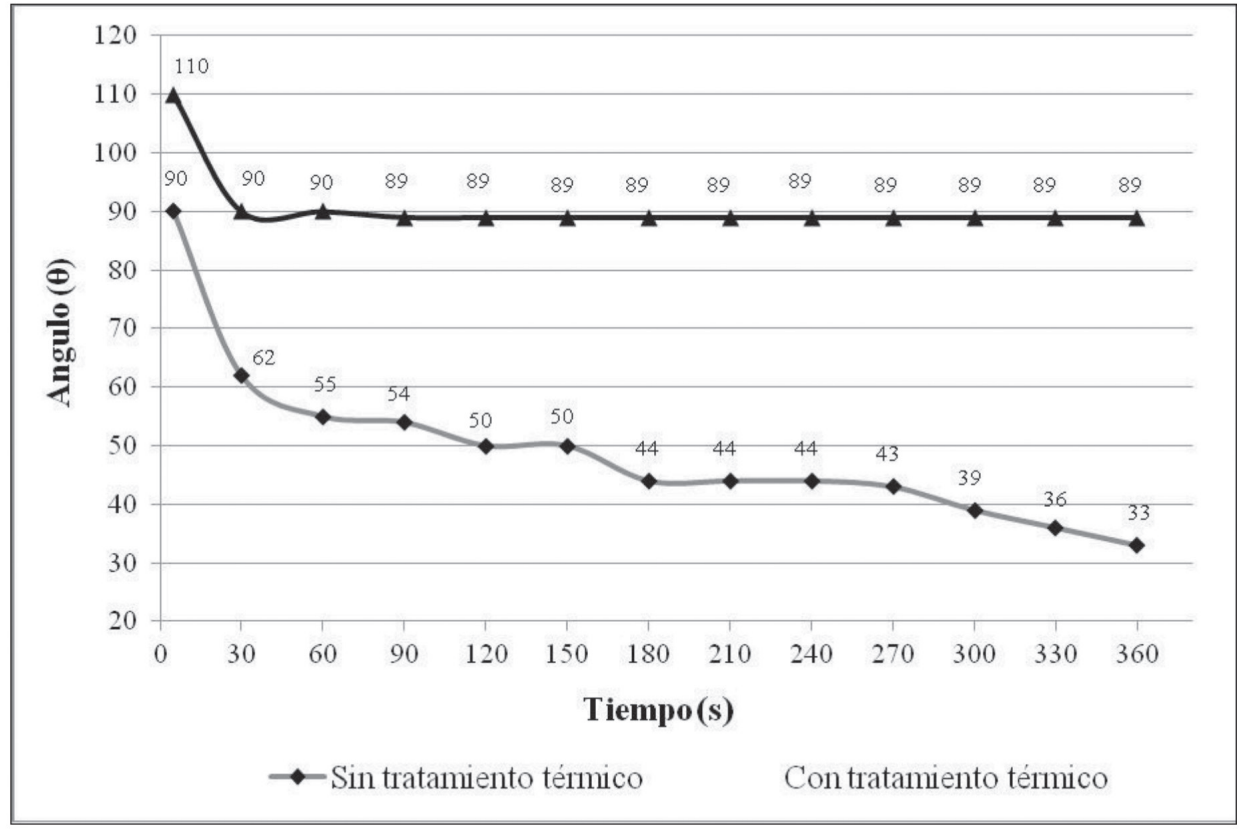

Figura 2. Valores del ángulo medido entre partículas de L. philippiana con una gota de ureaformaldehído ( $65 \%$ de sólidos) antes y después del tratamiento térmico.

Se observa en la figura 2 que los ángulos medidos en las partículas sin tratamiento disminuyen rápidamente y de forma permanente en el tiempo, lo que implica que la gota de adhesivo es absorbida por la madera. En el caso de las partículas tratadas, transcurridos 30 segundos el ángulo de contacto se estabiliza indicando que no hay absorción del adhesivo.

Hakkou et al. (2005) afirman que a temperaturas de tratamiento térmico entre 130 a $160{ }^{\circ} \mathrm{C}$ se reduce la humectabilidad de la madera, y que no se requiere de temperaturas más altas para modificar la propiedad hidrofílica de la madera. Los ángulos de contacto determinados en las partículas de L. philippiana tratadas térmicamente son similares con los reportados por Hakkou et al. (2005) después de un tratamiento en atmósfera de nitrógeno en madera de Fagus sylvatica. 
Sin embargo, Awoyemi et al. (2009a) informaron de un aumento de la humectabilidad con agua en madera de Picea marina y Pinus ponderosa después de un tratamiento térmico en aceite de soya caliente. La diferencia entre los resultados del presente estudio y los reportados por Awoyemi et al. (2009a) podría atribuirse a diferencias en la constitución química de las especies, los tratamientos aplicados (medio, temperatura y presión), y el líquido empleado para medir la humectabilidad.

Desde un punto de vista práctico, la disminución de la humectabilidad provocada por el tratamiento térmico, puede afectar la distribución del adhesivo para producir tableros, y también tener un efecto negativo en el pintado de los mismos.

Propiedades y características químicas antes y después del tratamiento

Los valores promedios de las diferentes determinaciones químicas en las partículas de L. philippiana se presentan en la tabla 2.

Tabla 2. Propiedades y características químicas de partículas de L. philippiana antes y después de un tratamiento térmico $\left(150^{\circ} \mathrm{C} / 90 \mathrm{~min}\right)$.

\begin{tabular}{|c|c|c|c|}
\hline \multicolumn{2}{|c|}{ Propiedad } & \multirow{2}{*}{$\begin{array}{c}\text { Partículas sin } \\
\text { tratamiento }\end{array}$} & \multirow{2}{*}{$\begin{array}{c}\text { Partículas con } \\
\text { tratamiento }\end{array}$} \\
\hline \multirow{4}{*}{$\begin{array}{c}\text { Extraíbles } \\
\text { (Promedio \% BMS) }\end{array}$} & Agua fría & & \\
\hline & Agua caliente & 1,2 & 4,6 \\
\hline & $\begin{array}{l}1 \% \text { de hidróxido de sodio } \\
(0,25 \mathrm{~N})\end{array}$ & 12,1 & 20,5 \\
\hline & Etanol tolueno & 2,6 & 5,5 \\
\hline \multirow{3}{*}{$\begin{array}{c}\text { Polímeros de la } \\
\text { madera (\%BMSLE) }\end{array}$} & Celulosa & 53,3 & 59,0 \\
\hline & Holocelulosa & 63,0 & 62,8 \\
\hline & Lignina & 29,5 & 30,4 \\
\hline \multirow[b]{3}{*}{ Valor de $\mathrm{pH}$} & Extraíbles en agua fría & 6,1 & 4,8 \\
\hline & Extraíbles en agua caliente & 5,8 & 4,2 \\
\hline & $\begin{array}{l}\text { Filtrado de una solución } \\
\text { de aserrín con agua luego } \\
\text { de agitar por } 4 \mathrm{~h} \text {. }\end{array}$ & 6,7 & 4,8 \\
\hline Ácidos volátiles (\%) & \multirow[b]{2}{*}{ Ácido como ácido acético } & 0,5 & 0,7 \\
\hline $\begin{array}{l}\text { Ácidos solubles en } \\
\text { agua }(\%)\end{array}$ & & 0,2 & 0,5 \\
\hline $\begin{array}{l}\text { Capacidad tampón } \\
\mathrm{NaOH} 0,01 \mathrm{~mol} / 1 \\
(\mathrm{mmol} / 100 \mathrm{~g} \text { madera }) \\
\end{array}$ & $\begin{array}{l}\begin{array}{l}\text { Solución de } \\
\text { consumida }\end{array} \\
\text { NaOH }\end{array}$ & 18,14 & 151,66 \\
\hline
\end{tabular}

BMS: Base a Madera Seca.

BMSLE: Base a Madera Seca Libre de Extraíbles, de acuerdo a la norma TAPPI 264 cm-97. 


\section{-Extraíbles}

En la tabla 2, se entregan los valores promedios de extraíbles en diferentes solventes, expresados como porcentaje en base a madera seca. Se encontró que en las partículas tratadas térmicamente el porcentaje de extraíbles en agua fría y en agua caliente cuadruplicó al de las partículas sin tratamiento. Resultados similares fueron encontrados por Esteves et al. (2011), quienes encontraron en madera de Pinus pinaster, un aumento del porcentaje de extraíbles en agua de $2,8 \%$ a $4,9 \%$, luego de ser tratadas a $190{ }^{\circ} \mathrm{C}$ por 2 y 8 horas.

El porcentaje de extraíbles en soda al $1 \%$ aumentó un $70,0 \%$ en las partículas tratadas térmicamente. Resultados similares fueron informados por Niemz et al. (2004) en madera de Eucalyptus nitens, donde se produjeron aumento de los extraíbles en soda de $13,3 \%$ a $33,9 \%$ a $150{ }^{\circ} \mathrm{C}$ por $60 \mathrm{~min}$.

Al extraer las partículas de L. philippiana en etanol tolueno se determinó un aumento de un $100 \%$ en el porcentaje de extraíbles al tratar las partículas a $150{ }^{\circ} \mathrm{C}$ por $90 \mathrm{~min}$. La tendencia a aumentar el porcentaje promedio de los extraíbles en etanol tolueno coincide con las determinaciones realizadas por Niemz et al. (2010), en madera de Fagus sylvatica, Pseudotsuga menziessi, Pinus sylvestris y Picea abies. La misma tendencia es informada por Esteves et al. (2011) en Pinus pinaster.

Según los resultados obtenidos en los distintos ensayos, las partículas tratadas térmicamente presentan un mayor porcentaje de extraíbles en agua fría, agua caliente, $1 \%$ de hidróxido de sodio $(0,25 \mathrm{~N})$ y etanol tolueno, que las partículas no tratadas. Los cambios en el contenido de extraíbles reflejan un cambio de los principales componentes químicos de la madera. El aumento de los extraíbles en las partículas tratadas térmicamente se debe a que la temperatura degrada las hemicelulosas, fraccionando las cadenas moleculares, y son extraídas por los solventes empleados.

El aumento del porcentaje de extraíbles probablemente afectará la resistencia de los tableros fabricados con partículas tratadas, debido a que durante el tratamiento térmico aumenta la cantidad de extraíbles hidrófobos, los cuales podrían impedir una buena unión de la madera con el adhesivo.

\section{- Celulosa, hemicelulosa y lignina}

En general, los valores obtenidos en las partículas no tratadas son coincidentes con los determinados por otros autores. (Urzúa et al. 1982). Los cambios registrados en partículas tratadas se deben al efecto que tiene tanto la temperatura como la presión aplicadas. En las partículas de L. philippiana tratadas, el porcentaje de celulosa aumentó un 5,7\%, con respecto a las no tratadas. De acuerdo a Kollmann y Fengel (1965), la celulosa sufre cambios significativos a partir de tratamientos con $180^{\circ} \mathrm{C}$, siendo poco alterada con temperaturas inferiores. El incremento del porcentaje de la celulosa presentado en la tabla 2, no se debe a un incremento en el contenido de celulosa, sino más bien a la relativa mayor degradación de las hemicelulosas, que hace cambiar la participación porcentual de los compuestos (Esteves et al. 2011). Fengel (1967) determinó cambios en la hemicelulosa a partir de los $150{ }^{\circ} \mathrm{C}$. Nguila et al. (2007), determinaron una disminución del porcentaje de holocelulosa en madera de Fagus sylvatica y en Pinus sylvestris al tratar con $240{ }^{\circ} \mathrm{C}$ en atmósfera de nitrógeno. Así también, Niemz et al. (2010) después de un tratamiento térmico a $180-200{ }^{\circ} \mathrm{C}$ por 1 a 4 horas en un ambiente de nitrógeno con una presión de 1000 $\mathrm{kPa}$, reportaron una disminución de la hemicelulosa en madera de Fagus sylvatica de $13,6 \%$ a 10,4\% y 5,6\% y de Pseudotsuga menziessi de 17,2\% a 13,3\% y 4,9\%. Los resultados de Niemz et al. (2010), con presión y tiempo mayores a los del presente estudio, son similares a los obtenidos con L. philippiana.

En el caso del presente estudio la degradación de las hemicelulosas es alta, de aproximadamente 7\% (Holocelulosa - Celulosa), lo que se debe al efecto de la temperatura y la presión generada en el autoclave. Esta despolimerización de la hemicelulosa se ve reflejada en un aumento de la participación de celulosa y un aumento general de los compuestos extraíbles en los diferentes solventes estudiados. 
Los porcentajes de lignina determinados en esta investigación no muestran diferencias entre los tratamientos (tabla 2). Esteves et al. (2011) reportaron un incremento máximo de 5,4\% de lignina luego de aumentar la temperatura y el tiempo de tratamiento a niveles significativamente más altos que los empleados en el presente estudio (190 a $210^{\circ} \mathrm{C}$ y 2 a 12 horas). Debe considerarse que durante el tratamiento en autoclave se generó una presión ( $\left.430 \mathrm{kPa}\right)$ que podría tener un efecto en la tasa de degradación de la madera.

\section{- Valor $\mathrm{pH}$}

Los valores de $\mathrm{pH}$ determinados en los extractos obtenidos en agua fría y caliente, así como los obtenidos en el filtrado de una solución de aserrín con agua, se presentan en la tabla 2. Los valores de $\mathrm{pH}$ medidos en L. philippiana sin tratamiento son similares a los obtenidos por otros autores en la misma especie (Poblete y Pinto 1993). El pH de las partículas con tratamiento térmico es considerablemente más ácido que el de partículas no tratadas. La reducción del valor de $\mathrm{pH}$ en las partículas de L. philippiana tratadas a $150{ }^{\circ} \mathrm{C}$, coincide con los resultados reportados por Awoyemi et al. (2009b) en madera de Pinus sylvestris $\left(160-200^{\circ} \mathrm{C}\right)$ y con los de Niemz et al. (2010) en madera de Fagus sylvatica, Pseudotsuga menziessi, Pinus sylvestris y Picea abies $\left(180-200{ }^{\circ} \mathrm{C}\right)$.

La acidificación que se produce en la madera, se debe a la acción de la temperatura sobre las hemicelulosas que son descompuestas, separación de los grupos acetilo, aumentando la cantidad de ácido acético y fórmico (Poblete y Roffael 1985).

Considerando los resultados y conclusiones de Poblete y Peredo (1990) y Pinto y Poblete (1992) respecto de las dificultades encontradas con L. philippiana al producir tableros con ureaformaldehído, la acidificación de las partículas tratadas permite deducir que el tratamiento térmico de la madera de $L$. philippiana es conveniente, puesto que facilitará el fraguado de la ureaformaldehído en la fabricación de tableros de partículas con esta especie.

\section{- Ácidos volátiles y solubles en agua}

La cantidad de ácidos volátiles y solubles en agua en las partículas con y sin tratamiento térmico se presentan en la tabla 2. La acidificación que se detecta en la madera de L. philippiana, por la disminución del valor de $\mathrm{pH}$, también se ve reflejada en un aumento de los porcentajes de ácidos volátiles y solubles en agua. Los valores encontrados, concuerdan con los determinados por Poblete (1983) en astillas de Fagus sylvatica y Picea abies, luego de un secado a $100{ }^{\circ} \mathrm{C}$ por 16 horas. En este estudio, el tratamiento a $150{ }^{\circ} \mathrm{C}$ por 90 minutos provocó un aumento de ácidos volátiles de un $20 \%$ en las partículas de L. philippiana. En el caso de los ácidos solubles en agua el porcentaje de estos compuestos aumentó a más del doble.

El aumento del porcentaje de ácidos volátiles y solubles en agua, determinado como ácido acético, explica el cambio de $\mathrm{pH}$ con el tratamiento, lo que, considerando los resultados de Poblete y Peredo (1990) y Pinto y Poblete (1992), facilitará el fraguado del adhesivo ureaformaldehído en el ambiente de $\mathrm{pH}$ desfavorable que provoca $L$. philippiana, siendo por lo tanto, beneficioso en la fabricación de tableros.

\section{- Capacidad tampón}

La determinación de la cantidad de hidróxido de sodio consumido es una forma indirecta de determinar la capacidad tampón de la madera. La cantidad de $\mathrm{NaOH} 0,01 \mathrm{~mol} / \mathrm{l}$, agregado en mmol/100g de madera hasta alcanzar un valor de $\mathrm{pH} 7$ representa la capacidad tampón de las partículas. Estos valores se detallan en la tabla 2. Los resultados comprueban la existencia de una importante diferencia en la característica de los extraíbles de la madera antes y después del tratamiento térmico. La disminución de la alcalinidad de las partículas al someterlas al tratamiento, da como resultado un aumento significativo en el consumo de $\mathrm{NaOH}$ de las mismas, con una capacidad de equilibrio de ionización (capacidad tampón) mucho mayor. Este efecto es determinado por los extraíbles de la madera. La disminución de la alcalinidad es muy importante para el proceso de encolado y fraguado, ya que, permite una mejor adherencia de la ureaformaldehído a las partículas y por lo tanto debiera aumentar las propiedades mecánicas de la unión. 


\section{CONCLUSIONES}

En las partículas de L. philippiana sometidas a un tratamiento térmico a $150{ }^{\circ} \mathrm{C}$ por 90 minutos, se produjo un cambio de color de amarillo pálido a café, así como una menor capacidad de humectabilidad. Se detectó un aumento de los porcentajes de extraíbles en agua fría, agua caliente, $1 \%$ de hidróxido de sodio $(0,25 \mathrm{~N})$ y etanol tolueno. El porcentaje de celulosa y lignina aumentaron 5,7\% y 0,9\%, respectivamente. El valor de $\mathrm{pH}$ se redujo en promedio de 6,2 a 4,6, mientras que el porcentaje de ácidos volátiles y solubles en agua, así como, la capacidad tampón aumentaron significativamente.

Las características químicas y físicas de las partículas de L. philippiana que fueron tratadas térmicamente, confirman que el tratamiento térmico de la madera de esta especie puede ser ventajoso previo a la fabricación de tableros de partículas, puesto que facilitaría el fraguado del adhesivo ureaformaldehído y la reacción de este con la madera. Sin embargo, el cambio de color puede ser una desventaja.

\section{BIBLIOGRAFÍA}

Awoyemi, L.; Cooper, P.; Ung, T. 2009a. In-treatment cooling during thermal modification of wood in soy oil medium: soy oil uptake, wettability, water uptake and swelling properties. Eur J Wood Prod 67 (4): 465-470.

Awoyemi, L.; Jarvis, E.; Hapca, A. 2009b. Effects of preboiling on the acidity and strength properties of heat-treated Wood. Wood Sci Technol 43 (1-2):97-103.

Bächle, H.; Zimmer, B.; Windeisen, E.; Wegener, G. 2010. Evaluation of thermally modified beech and spruce wood and their properties by FT-NIR spectroscopy. Wood Sci Technol 44 (3):421-433.

Bekhta, P.; Lecka, J.; Morze, Z. 2003. Short-term effect of the temperature on the bending strength of woodbased panels. Holz als Roh- und Werkstoff 61 (6):423-424.

Bekhta, P.; Marutzky, R. 2007. Bending strength and modulus of elasticity of particleboards at various temperatures. Holz Roh Werkst 65 (2):163-165.

Boonstra, M.; Pizzi, A.; Zomers, F.; Ohlmeyer, M.; Paul, W. 2006a. The effects of two stage heat treatment process on the properties of particleboard. Holz als Roh- und Werkstoff 64 (2):157-164.

Boonstra, B.; Rijsdijk, J.; Sander, C.; Kegel, E.; Tjeerdsma, B.; Militz, H.; Van Acker, J.; Stevens, M. 2006b. Microstructural and physical aspects of heat treated wood. Part 1. Softwoods. Maderas. Ciencia y tecnología $8(3): 193-208$.

Boonstra, M.; Tjeerdsma, B. 2006. Chemical analysis of heat treated softwoods. Holz als Roh- und Werkstoff 64 (3): 204-211.

Esteves, B.; Videira, R.; Pereira, H. 2011. Chemistry and ecotoxicity of heat treated pine wood extractives. Wood Sci Technol 45 (4):661-676.

Fengel, D. 1967. Über die Veränderungen des Holzes und Seiner Komponenten im Temperaturbereich bis 200 ${ }^{\circ} \mathrm{C}-$ Vierte Mitteilung: Das Verhalten der Cellulose im Fichtenholz bei thermischer Behandlung. Holz als Roh und Werkstoff 25 (3):102-110.

Fengel, D.; Wegener, G. 1983. Wood. Chemistry, Ultrastructure, Reactions. Walter de Gruyter. Berlin, Germany. $613 \mathrm{p}$. 
Figueroa, M.; Moraes, P. 2009. Comportamento da madeira a temperaturas elevadas. Ambiente Construído 9 (4): $57-174$

Giebeler, E. 1983. Dimensionsstabilisierung von Holz durch eine Feuchte/Wärme/Druck-Behandlung. Holz als Roh- und Werkstoff 41 (3): 87-94.

Hakkou, M.; Pétrissans, M.; Zoulalian, A.; Gérardin, P. 2005. Investigation of wood wettability changes during heat treatment on the basis of chemical analysis. Polymer Degradation and Stability 89 (1): 1-5.

Haygreen, J.; Bowyer, J. 1982. Forest products and Wood science. First edition. The Iowa State University press. Iowa, United Sates. 495p.

Hessler, L.; Merola, G. 1949. Determination of Cellulose in Cotton and Cordage Fiber. Analytical Chemistry 21 (6):695-698.

Kartal, S.; Hwang, W.J.; Imamura, Y. 2007. Water absorption of boron-treated and heat-modified wood. $J$ Wood Sci 53 (5): 454-457.

Kocaefe, D.; Chaudhry, B.; Poncsak, S.; Bouazara, M.; Pichette, A. 2007. Thermogravimetric study of high temperature treatment of aspen: effect of treatment parameters on weight loss and mechanical properties. $J$ Mater Sci 42 (3): 854-866.

Kocaefe, D.; Poncsak, S.; Tang, J.; Bouazara, M. 2010. Effect of heat treatment on the mechanical properties of North American jack pine: thermogravimetric study. J Mater Sci 45 (3): 681-687.

Kollmann, F.; Fengel, D. 1965. Änderungen der chemischen Zusammensetzung von Holz durch thermische Behandlung. Holz als Roh- und Werskstoff 23 (12): 461-468.

Martin-Sampedro, R.; Capanema, E.; Hoeger, I.; Villar, J.; Rojas, O. 2011a. Lignin Changes after Steam Explosion and Laccase-Mediator Treatment of Eucalyptus Wood Chips. J Agric Food Chem 59 (16): 8761-8769.

Martín-Sampedro, R.; Martín, J.; Eugenio, M.; Revilla, E.; Villar, J. 2011b. Steam explosion treatment of Eucalyptus globulus wood: influence of operational conditions on chemical and structural modifications. Bioresources 6 (4): 4922-4935.

Metsä-Kortelainen, S.; Viitanen, V. 2012. Wettability of sapwood and heartwood of thermally modified Norway spruce and Scots pine. Eur J Wood Prod 70 (1-3):135-139.

Munsell. 1994. Soil color charts. Revised edition. Gretag Macbeth. New Windsor, United States.

Nguila, G.; Petrissans, M.; Gerardin, P. 2007. Chemical reactivity of heat treated wood. Wood Sci Technol $41(2): 157-168$.

Niemz, P.; Hofmann, T.; Rétfalvi, T. 2010. Investigation of chemical changes in the structure of thermally modified Wood. Maderas. Ciencia y tecnología 12 (2):69-78.

Niemz, P.; Mariani, S.; Torres, M. 2004. Einfluss der hydrothermischen Behandlung von Picea abies (L.) Karten und Eucalyptus nitens (Deane \& Maiden) Maiden auf die chemische Zusammensetzung des Holzes. Schweiz Z Forstwes 155 (12): 544-547. 
Petric, M.; Knehtl, B.; Krause, A.; Militz, H.; Paylic, M.; Pétrissans, M.; Rapp, A.; Tomazic, M.; Welzbacher, C.; Gérardin, P. 2007. Wettability of waterborne coatings on chemically and thermally modified pine wood. Journal of Coatings Technology and Research 4 (2): 203-206.

Pinto, A.; Poblete, H. 1992. Efecto del catalizador en las propiedades de tableros de partículas con madera de tepa. Ciencia e Investigación Forestal 6 (2): 259-279.

Poblete, H. 1983. Veränderungen in Holzspänen Während der Trocknung und des Pressens zu Holzspanplatten. Dissertation zur Doktorgrades. Georg August Universität zu Göttingen. 157 pp.

Poblete, H.; Niemz, P.; Baechle, F.; Schanack, F. 2005. Investigation on Hydrothermal Treatment of Pinus radiata D. Don. Wood Research 50 (1): 33-41.

Poblete, H.; Peredo, M. 1990. Tableros de desechos del debobinado de especies chilenas. Bosque 11 (2): 45-58.

Poblete, H.; Pinto, A. 1993. Avances sobre el efecto del catalizador en el fraguado de ureaformaldehído en tableros de L. philippiana. Bosque 14 (1): 55-61.

Poblete, H.; Roffael, E. 1985. Über chemische Veränderungen in Holzspänen bei der Herstellung von HarnstoffFormaldehydharz-gebundenen Spanplatten. Holz als Roh- und Werkstoff 43 (2):57-62.

Rodríguez, L. 1978. Métodos de análisis. Empleados en la industria del papel. Primera edición. Universidad Industrial de Santander. Bucaramanga, Colombia. 156 p.

Roffael, E.; Poblete, H.; Torres, M. 2000. Über die Acidität von Kern - und Splintholz der Kastanie (Castanea sativa) aus Chile. Holz als Roh- und Werkstoff 58 (1-2):120-122.

Schaffer, E. 1973. Effect of Pyrolytic Temperatures on the Longitudinal Strength of Dry Douglas-Fir. Journal of Testing and Evaluation 1(4): 319-329.

Sundqvist, B.; Morén, T. 2002. The influence of wood polymers and extractives on wood colour induced by hydrothermal treatment. Holz als Roh- und Werkstoff 60 (5): 375-376.

TAPPI. Technical Assotiation of the Pulp and Paper Industry. 2000. TAPPI Test Methods $2000-2001$. TAPPI Press. Atlanta, United States.

Tjeerdsma, B.; Boonstra, M.; Pizzi, A.; Tekely, P.; Militz, H. 1998. Characterization of thermally modified Wood: molecular reasons for Wood performance improvement. Holz als Roh- und Werkstoff 56 (3):149-153.

Urzúa, D.; Aguilar, F.; Diaz-vaz, J.; Guerrero, G.; Peredo, L.; Vergara, C, Inzunza, L.; Torres, M. 1982. Utilización silvoagropecuaria de terrenos de Ñadi. Aspectos tecnológicos de las maderas de Ñadi. Informe de convenio $\mathrm{N}^{0}$ 54. Universidad Austral de Chile. Valdivia, Chile. 156p.

Yao, C.; Yongming, F.; Jianmin, G.; Houkun, L. 2012. Coloring characteristics of in situ lignin during heat treatment. Wood Sci Technol 46 (1-3):33-40.

Yongming, F.; Jianmin, G.; Yao, C. 2010. Colour responses of black locust (Robinia pseudoacacia L.) to solvent extraction and heat treatment. Wood Sci Technol 44 (4):667-678. 